\title{
ERstudios EGerenciales
}

Journal of Management and Economics for Iberoamerica

Artículo de investigación

\section{Implicaciones de la COVID-19 sobre el marketing de servicios educativos: un estudio desde las motivaciones y estados de ánimo de universitarios en Colombia}

\author{
Juan Camilo Mejía* \\ Profesor, Programa de Comercio Internacional y Mercadeo, Universidad de Nariño, Pasto, Colombia. \\ Candidato a doctor en Marketing, Universidad de Valencia, Valencia, España. \\ mejuanca@alumni.uv.es
}

Diego De la Rosa-Salazar

Profesor, Programa de Comercio Internacional y Mercadeo, Universidad de Nariño, Pasto, Colombia. diegorosa@udenar.edu.co

Hernán Darío Huertas-Moreno

Profesor, Programa de Mercadeo, Universidad Mariana, Pasto, Colombia.

hhuertas@umariana.edu.co

\section{Resumen}

Este estudio analizó las implicaciones que tiene la pandemia en la aplicación del marketing de servicios educativos en instituciones de educación superior en Colombia; en particular, se revisaron las implicaciones asociadas con las motivaciones por estudiar y los estados de ánimo presentes en los estudiantes. Para ello, se realizó un estudio empírico en cinco universidades, que recogió las opiniones de 695 estudiantes. Primero, se realizó un análisis factorial exploratorio y luego un análisis clúster. Los resultados evidenciaron las diferentes dificultades que se presentaron en la aplicación del marketing, desde la perspectiva estudiantil. Como resultado, el estudio sugiere algunas recomendaciones para aplicar al marketing educativo bajo el contexto de la pandemia.

Palabras clave: COVID-19; marketing de servicios educativos; motivaciones; estados de ánimo.

Implications of COVID-19 on the marketing of educational services: A study from the motivations and moods of university students in Colombia

\section{Abstract}

This study analyzed the implications of the pandemic on the application of marketing of educational services in higher education institutions in Colombia; in particular, the implications associated with the motivations for studying and the moods present in the students were reviewed. For this, an empirical study in five universities, which collected the opinions of 695 students, was carried out. First, exploratory factor analysis was performed, and then, a cluster analysis. The results showed the different difficulties in the application of marketing, from the student perspective. As a result, the study suggests some recommendations to apply to educational marketing in the context of the pandemic.

Keywords: COVID-19; marketing of educational services; motivations; moods.

Implicações do COVID-19 no marketing de serviços educacionais: um estudo a partir das motivações e estados de ânimo de estudantes universitários na Colômbia

\section{Resumo}

Este estudo analisou as implicacões da pandemia na aplicação do marketing de servicos educacionais em instituicões de ensino superior na Colômbia; em particular, foram revistas as implićações associadas às motivações para estudar e aos estados de ânimo presentes nos alunos. Para isso, foi realizado um estudo empírico em seis universidades, que coletou a opinião de 695 alunos. Primeiramente, foi realizada uma análise fatorial exploratória e, em seguida, uma análise de cluster. Os resultados mostraram as diferentes dificuldades que surgiram na aplicação do marketing, na perspectiva dos alunos. Como resultado, o estudo sugere algumas recomendações a serem aplicadas ao marketing educacional no contexto da pandemia.

Palavras-chave: COVID-19; marketing de serviços educacionais; motivações; estados de ânimo.

* Autor para dirigir correspondencia.

Clasificación JEL: M3; M31; M39.

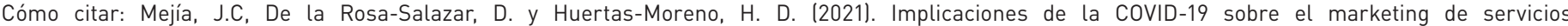

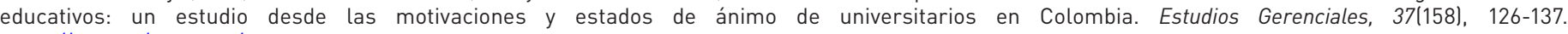
https://doi.org/10.18046/j.estger.2021.158.4271

DOI: https://doi.org/10.18046/j.estger.2021.158.4271

Recibido: 2-sep-2020

Aceptado: 18-ene-2021

Publicado: 31-mar-2021

(C) 2021 Universidad ICESI. Published by Universidad Icesi, Colombia

This is an open access article under the CC BY license (http://creativecommons.org/licenses/by/4.0/) 


\section{Introducción}

La pandemia generada por COVID-19 Icorona virus disease-2019) generó grandes cambios en diferentes ámbitos: económico, sanitario, social y educativo (Sohrabi et al., 2020). Como los pronósticos acerca del desarrollo de una vacuna eran inciertos, la estrategia principal disponible para prevenir la rápida propagación del virus consistió en el distanciamiento social (Del Rio y Malani, 2020). Esta situación impide que las personas se reúnan en espacios donde los riesgos de contagio aumenten, razón por la cual las universidades se vieron obligadas a cerrar sus instalaciones, atendiendo a normas de distanciamiento impuestas por los gobiernos (Wang, Cheng, Yue y McAleer, 2020). En palabras de Audrey Azoulay, directora General de la Organización de las Naciones Unidas para la Educación, la Ciencia y la Cultura (Unesco, 2020a): “Nunca antes habíamos presenciado una interrupción educativa a una escala tan grande" (párr. 3).

Durante la mitad del primer semestre del año 2020, la mayoría de las universidades en Colombia se vieron obligadas a trasladarse de una modalidad presencial a una mediada por las tecnologías. Este abrupto cambio se dio sin tener en cuenta una debida planificación y sin la aplicación de inversiones significativas para hacerlo de la mejor manera (Crawford et al., 2020). Debido a esto, toda la comunidad educativa vivió un cambio sin precedentes, que influyó en la entrega de este servicio de forma tradicional. En tal contexto, la presente investigación describe y analiza el impacto de la pandemia sobre el marketing en las universidades, para entender aquellas actividades que desde esta área se dejaron de hacer, se hacen y se harán bajo este contexto. Con este objetivo en mente se revisan las opiniones de estudiantes universitarios de Colombia sobre sus motivaciones y estados de ánimo durante la pandemia. También, en atención a la revisión de literatura que se ha hecho hasta el momento, se comentan interesantes hallazgos para trabajar el tema propuesto (Charles, 2020; Dorn, Hancock, Sarakatsannis y Viruleg, 2020; García-Rey, 2020; Papandrea, 2020; Speth et al., 2020; Ting, Carin, Dzau y Wong, 2020; Wang et al., 2020; Zhang et al., 2020; entre otros).

Este estudio utiliza la técnica de análisis factorial exploratorio para simplificar la información obtenida y hacerla interpretable de una manera más fácil, para luego realizar con estos resultados un análisis de clúster (Hair, Anderson, Tatham y Black, 1999). Además, ofrece información relevante que sirve como referente para la toma de decisiones estratégicas de marketing en instituciones de educación superior.

La organización de este trabajo presenta una revisión de literatura, la metodología implementada, los resultados obtenidos y las conclusiones principales en las que se presentan recomendaciones, dificultades en la investigación y futuras líneas de investigación.

\section{Revisión de literatura}

2.1 Impacto de la pandemia por COVID-19 en el marketing de servicios educativos

La American Marketing Association (AMA), en el año 2017, definió el marketing como "actividad, conjunto de instituciones y procesos para crear, comunicar, entregar e intercambiar ofertas que tienen valor para los consumidores, clientes, socios y la sociedad en general" (AMA, 2017, párr. 2, traducción propia). Desde el enfoque de marketing convencional, McCarthy (1960) propone la mezcla de marketing, más conocido como marketing mix, para que sea aplicado en el intercambio de bienes físicos y transacciones como su principal razón; este enfoque es conocido comúnmente como las 4P. Sin embargo, a medida que los servicios ganaron más peso en la economía, los investigadores se interesaron en estudiar el marketing en diversas áreas. Vargo y Lusch (2004) definen el servicio como "la aplicación de competencias especializadas (conocimientos y habilidades), a través de hechos, procesos y actuaciones en beneficio de otra entidad o de la propia entidad" (p. 2).

A partir de la década de 1970, se llegó a la conclusión de que los axiomas clásicos del marketing no podían proporcionar una comprensión suficiente de los servicios y comenzaron a desarrollar una teoría del marketing de servicios basada en sus características distintivas (Grönroos, 1982), bajo el entendido de lo importante que son las interacciones entre consumidores (usuarios) y vendedores (proveedores de servicios), que dan lugar al marketing de servicios. Acerca de la educación, Shostack (1982) sostiene que esta tiene una alta dominancia del servicio y para demostrarlo construyó una escala comparativa de dominancia que ordena las entidades de acuerdo con su composición general.

La educación como servicio puro se caracteriza desde el marketing, principalmente, por las siguientes características: 1) intangible, es decir, no se puede percibir antes, y en ocasiones, ni durante ni después de su prestación, en aspectos claves como la adquisición y la distribución; 2) inseparable, respecto a que la producción y el uso no pueden separarse, es el caso de la relación estudiante-docente en el proceso de enseñanza; 3) variable, como consecuencia inevitable de la producción y el consumo simultáneos, la calidad del servicio puede variar según quién, cuándo y cómo se brinde, por lo que es imposible estandarizarlo, dependiendo en mayor medida de cada estudiante para el caso educativo; y 4) perecedera, es decir, no se puede almacenar para su posterior venta o uso, ya que el proceso de enseñanza no es almacenable; si no hay matrículas, eso representa una pérdida para ese periodo (Rushton y Carson, 1985).

En el ámbito de los servicios, y específicamente para la educación, los autores proponen el modelo de las 7P. En este se tiene en cuenta, en primer lugar, la propuesta de McCarthy (1960) para la mezcla de marketing tradicional, es decir, las 4P: 1P) producto, entendido como el servicio 
educativo completo; 2P) precio, entendido como el intercambio económico por el servicio recibido, independiente de si es el Gobierno o el estudiante quien realiza el pago; 3P) plaza, la que refiere al canal de entrega del servicio educativo, que puede ser presencial, a distancia o virtual; 4P) promoción, entendida como la mezcla de comunicaciones para difundir la información del proyecto educativo o de la oferta en cuestión. En segundo lugar, se tiene en cuenta la propuesta de Booms y Bitner (1981), quienes agregan las $P$ restantes: $5 P$ I personas, que se refiere a la apariencia y comportamiento del personal de servicio administrativo, operativo o académico, según sea el caso; 6P) presencia o evidencia física, que es la tangibilización del servicio, en el que se encuentran las instalaciones, el equipamiento, la vestimenta, entre otras; y 7) proceso, que comprende la relación enseñanza-aprendizaje, la organización de la institución, la evaluación, entre otros.

En algunos círculos, el concepto de marketing educativo se observa de manera sospechosa debido a las implicaciones que esta perspectiva pueda tener en la mercantilización de la educación y sus consecuencias éticas. Sin embargo, para los autores del artículo, de aplicarse correctamente el marketing educativo, resulta más beneficioso que perjudicial y es claramente necesario para centros educativos de todo tipo, pues permite proveer una oferta de valor interesante y relevante para los actores asociados a la educación (Harvey, 1996). Por ello, las instituciones han aprovechado el marketing en un intento por lograr sus objetivos organizacionales, al tener unos procesos establecidos de consecución de estudiantes y de entrega de valor.

Sin embargo, bajo los efectos de la pandemia en el servicio educativo, el Ministerio de Educación Nacional (MEN) de Colombia se acogió a la Resolución 385, emitida por el Ministerio de Salud y Protección Social (2020), que limita el contacto social como una medida para enfrentar la crisis sanitaria. Dicho aislamiento prohíbe a toda la comunidad académica de cualquier nivel, ingresar a los espacios donde normalmente se efectúan las clases. A nivel mundial, la Unesco (2020a) estima que el $87 \%$ de los estudiantes han suspendido su educación en modalidad presencial.

Para algunas universidades que antes de la pandemia COVID-19 ya fundamentaban su sistema de enseñanza en modalidad virtual o a distancia, esta situación no surtió cambios dramáticos (García-Rey, 2020). Sin embargo, para el año 2018, según un boletín de la Asociación Colombiana de Universidades (ASCUN, 2020, p. 6) de 2.067 .925 matrículas en programas académicos, 185.176 se realizaron en programas virtuales, lo que representa únicamente un $8,95 \%$ de ese total. Estos datos para Colombia dejan a la mayoría de las instituciones de educación superior bajo un complejo y difícil panorama para cumplir esa propuesta de valor previamente soportada por la presencialidad. En este marco, se presentan las perspectivas que algunos autores han desarrollado sobre los impactos que ha tenido la pandemia por COVID-19 en el servicio educativo, enfocados en las 7P.

\subsubsection{Impacto sobre el producto}

Sobre el concepto de Vargo y Lusch $(2004$, p. 2) y la primera P como el servicio educativo global, la decisión tomada por el Ministerio de Salud y Protección Social (2020) produce una transición inesperada de un conjunto de atributos que atendían a una necesidad latente, y a un deseo por aprender de manera presencial, a uno que se maneja mediante el uso de tecnologías. En este sentido, resulta difícil para las instituciones, primero, garantizar la puesta en marcha de esta modalidad; segundo, adquirir la capacidad para controlar aspectos de calidad relacionados con este servicio; tercero, proveer soluciones relevantes para los estudiantes, docentes y administrativos; y cuarto, genera un desconocimiento acerca de la vulnerabilidad en los hogares y contextos que no cuentan con las posibilidades para desarrollar esta modalidad remota de aprendizaje, además de ignorar los efectos emocionales y psicológicos que pueden influir en el proceso de enseñanza-aprendizaje en esta pandemia (Cabrera, 2020).

\subsubsection{Impacto sobre el precio}

En esta variable el impacto no ha sido inmediato, sino que el golpe a los ingresos, debido a un posible aumento en la deserción, solo se puede medir bajo la confirmación de este supuesto en el periodo subsecuente. Desde la perspectiva económica, los estudiantes son los demandantes principales de este servicio, lo que implica que, ya sea el mismo usuario, algún familiar o el Gobierno quien pague, son los principales proveedores de ingresos de las instituciones. Cabe tener en cuenta, además, que por el confinamiento la economía de los hogares se transformó en una economía de supervivencia, a lo que se suma que gran parte de los colombianos han perdido su trabajo y el poco apoyo gubernamental para cumplir con ciertos gastos, entre ellos la educación. En este contexto, es un desafío garantizar que la mayoría de los estudiantes vuelvan a las universidades, independiente de la modalidad en la cual se desarrolle el servicio (Goodell, 2020; Unesco, 2020a); esto implica que la variable se analice para ser consecuente con dos cuestiones importantes. La primera tiene que ver con las prioridades en el gasto de los hogares $y$, la segunda, con las necesidades de las universidades de recibir ingresos para su mantenimiento y pagar sus obligaciones.

\subsubsection{Impacto sobre la plaza}

Con el cierre de los centros de estudio, se obligó a las universidades a utilizar herramientas, plataformas y recursos para establecer y abrir canales de apoyo, con el objetivo de terminar el semestre académico. Si bien estas herramientas no han sido las mejores para atender este suceso, las instituciones se han visto en la necesidad de realizar inversiones en adquisición y mejoras de este tipo (Wang et al., 2020). Por otra parte, la pandemia incentivó a instituciones más grandes e importantes a nivel mundial 
a crear y promover plataformas de enseñanza de uso masivo y sin costo, además de recursos de alta calidad que podrían significar un salto cualitativo para la producción de conocimiento a futuro (Hylén, 2006).

\subsubsection{Impacto sobre la promoción}

Desde la perspectiva de algunos autores, la promoción ha sido impactada positivamente gracias a la transición obligatoria hacia las tecnologías y la virtualidad, por lo que las instituciones debieron adaptar también sus contenidos al contexto, utilizar componentes dirigidos a lo emocional y consolidar el compromiso por mejorar las percepciones sobre este acontecimiento. Aunque es poca la evidencia empírica, se cree que lo digital está menos afectado que otros medios, ya que las personas en cuarentena pueden pasar más tiempo en internet; pero la investigación sobre los formatos más efectivos en estos tiempos aún no se ha realizado. En definitiva, todo lo relacionado con la promoción de las universidades fortaleció el conjunto de actividades que se desarrolló dentro y fuera de ellas (Taylor, 2020).

\subsubsection{Impacto sobre el personal}

Frente a este punto, día a día se llevan a cabo procesos de capacitación y motivación con todo el personal: administrativos, operativos, docentes e incluso estudiantes. Aun así, parar muchas universidades, se plantea un dilema entre implementar soluciones rápidas o analizar profundamente las necesidades de la comunidad académica. En ese sentido, ha sido evidente que la pandemia por COVID-19 presenta un gran reto en materia educativa, que ha debido enfrentar la mayoría de actores involucrados: desde estructurar programas virtuales; formar redes colaborativas; mejorar el trato y responder en tiempo real a los interrogantes por medio de las plataformas virtuales; dominar habilidades técnicas para el manejo de nuevas tecnologías; adaptarse a nuevas metodologías y formas de enseñanza-aprendizaje; hacer uso de nuevos programas y aplicaciones educativas; hasta cambiar el paradigma tradicional de educación presencial (Dorn et al., 2020; Toledo y Armas, 2020).

\subsubsection{Impacto sobre la evidencia física}

El principal cambio que la pandemia ha influido en el sistema educativo es el cambio de un modelo presencial tradicional a un modelo educativo mediado por tecnologías, en el cual se presentaron dificultades relacionadas con varios aspectos. En principio, se deben tener en cuenta las dificultades relacionadas con las necesidades tecnológicas propias del paso a la virtualidad. Según un informe de la Unesco (2020b): "La mitad del total de los alumnos -unos 826 millones de estudiantes- que no pueden asistir a la escuela debido a la pandemia de COVID-19, no tienen acceso a una computadora en el hogar y el $43 \%$ (706 millones) no tienen Internet en sus casas" (párr. 1).
En segundo lugar, se deben considerar las afectaciones físicas por mobiliario, pues los estudiantes deben estar mucho tiempo frente al computador. En tercer lugar, la educación virtual implica limitaciones de espacio y condiciones inadecuadas, como la falta de luz y ventilación de la habitación de estudio, que no se comparan con las del salón de clase normal. Por último, la competencia por los recursos del hogar, debido principalmente a que el confinamiento incluye a todos sus miembros y algunos de ellos demandan diferentes recursos para realizar sus actividades (Hernández, 2020).

\subsubsection{Impacto sobre los procesos}

Aún no se tiene información empírica acerca de las implicaciones sobre el proceso de enseñanza-aprendizaje. Algunos autores evidenciaron que durante la pandemia por COVID-19 los estudiantes se han motivado a estudiar en línea desde sus hogares, aunque se necesitan estudios futuros para confirmar los resultados. Contrario a lo que se pensaría, usar el estudio en línea de manera más efectiva aumentaría los niveles de concentración pormás tiempo (Zhang et al., 2020). Sin embargo, la poca experiencia en manejo educativo para momentos de crisis, como el actual, ha demostrado que la virtualidad posee ciertas ventajas que poco a poco cada actor va descubriendo, aunque existan deficiencias en el proceso. Por lo tanto, esta modalidad ha permitido un perfeccionamiento progresivo de las actividades relativas a la autonomía en el aprendizaje, maximización de recursos creativos y adaptación al cambio (Idrovo, 2020).

\subsection{Motivaciones}

Desde un enfoque de relación aprendizaje-comportamiento, la motivación se define como un impulso que mueve a un individuo a la acción siempre y cuando tal estímulo posea una fuerza determinada (Miller y Dollard, 1941; Morgan, 1943). La motivación refiere a propósitos conscientes o inconscientes que hacen parte de un todo integrado y organizado, lo que implica que un individuo no puede motivar una parte de sí mismo, sino el conjunto que este representa (Maslow, 1954).

La motivación se divide en dos dimensiones. La primera es la motivación intrínseca, que se define como la realización de una actividad por la satisfacción propia, es decir que existe un deseo por actuar debido al desafío que conlleva, en lugar de hacerlo por estímulos, presiones o recompensas externas. Esta, a su vez, se divide en dos subdimensiones: al la motivación por conocer, que hace referencia a encontrar placer mientras se aprende o se comprende algo nuevo y b) la motivación por los logros, relacionada con la satisfacción que experimenta el individuo al alcanzar los objetivos propuestos (Deci y Ryan, 1985; Ryan y Deci, 2000).

La segunda dimensión de la motivación es la extrínseca, que se refiere a la participación del sujeto en diferentes actividades para conseguir recompensas, lo que implica 
que su conducta se dirija a un fin predefinido, aunque este no sea precisamente para su propio bien. En ella se tiene en cuenta el nivel de autonomía del sujeto para atender a una motivación en particular y el fin esperado (Ryan y Deci, 2000). Por ello, los autores también la dividen en subdimensiones: a) la regulación externa, que es la participación en una actividad para conseguir recompensas o evitar castigos y surge por la recepción de presiones externas; b) la regulación introyectada, que es un tipo de regulación interna que todavía es bastante controlada por otros para realizar acciones que permitan evitar la culpa o la ansiedad o para lograr mejoras del ego o del orgullo - en este caso, el individuo ya racionaliza sus acciones aunque sigue siendo por demanda externa y no es autodeterminada-; y cl la regulación identificada, en la que la persona se identifica con la importancia personal de un comportamiento y, por tanto, acepta su regulación como propia (Deci y Ryan, 1985).

Brophy (1983) sugirió el concepto de motivación del estudiante para aprender, según el cual, la motivación se puede ver como un rasgo general y un estado específico para aprender. En cuanto al rasgo general, se refiere a una disposición duradera a valorar el aprendizaje por sí mismo, a disfrutar del proceso y enorgullecerse con los resultados de las experiencias que implican la adquisición de conocimientos o el desarrollo de habilidades. En situaciones específicas, los estudiantes se involucran con determinación en las tareas del aula y tratan de dominar los conceptos o habilidades involucrados. Ellos se motivan para aprender no necesariamente bajo el concepto de placer o emoción, pero encuentran significativo y valioso los beneficios de hacerlo (Brophy, 1983).

El nivel de motivación sugiere proporcionalmente el nivel y la calidad de participación que probablemente mantenga el estudiante, y para explorar esta línea se asume que el estudiante evita su propio fracaso, convirtiendo esto en un patrón de motivación (Covington y Omelich, 1984). Con la pandemia por COVID-19, surgió un patrón diferente en el cual el estudiante disminuyó su nivel de participación y motivación, y empezó a preocuparse por el aspecto evaluativo. La evaluación se convierte en uno de los elementos fundamentales de la motivación del estudiante, y por esto es preciso orientarla hacia diferentes objetivos (Ames y Ames, 1984; Crawford et al., 2020; Ting et al., 2020).

\subsection{Estado de ánimo}

Los estados de ánimo se pueden determinar cuando el sistema cognitivo se mantiene en un modo emocional durante un período (Bagozzi, Gopinath y Nyer, 1999). Frijda (1986) afirma que los estados de ánimo son provocados, principalmente por secuelas de las emociones o por condiciones orgánicas-ambientales y también por los efectos secundarios de las actividades del sujeto. Por ello, es importante sugerir que el estado de ánimo puede afectar el proceso cognitivo de los individuos, e influye en procesos como retención y procesamiento de la información (Forgas y Bower, 1987).

La Organización Internacional del Trabajo (OIT) declaró que la pandemia generó cambios psicológicos que incluyen aumento de la ansiedad, baja motivación y pensamientos depresivos, debido principalmente a la amenaza de contagio, cantidad alarmante de enfermos, las cifras de mortandad y las pérdidas de tipo económico que esta causó (Papandrea, 2020). Según el informe de Papandrea (2020), los dos estados anímicos latentes en este acontecimiento fueron, primero, la ansiedad, definida como un estado de agitación, inquietud o zozobra del ánimo y que se puede evaluar en el individuo por medio de adjetivos como tenso, agitado, a punto de estallar, descontrolado, relajado, intranquilo, inquieto, nervioso; y, segundo, la depresión, conceptualizada como la pérdida del yo, desde una perspectiva interaccionista simbólica, en la que el yo se experimenta y construye a través de las relaciones sociales y se puede evaluar por adjetivos como infeliz, triste, abatido, desesperanzado, torpe, desanimado, solo, desdichado, deprimido, desesperado, desamparado, inútil, aterrorizado y culpable (Mead, 1934; Thyer, 1987; Papandrea, 2020).

Las alteraciones psicoemocionales de los estudiantes por efecto de la COVID-19 aún no se han caracterizado completamente. Sin embargo, Speth et al. (2020) asociaron los estados de ánimo deprimido y ansioso a esta pandemia. Por su parte, Charles (2020) brindó información sobre cómo la pandemia y la alteración asociada afectaron la salud mental y el bienestar de los adultos jóvenes, aunque no sean tan propensos a experimentar síntomas graves por este nuevo coronavirus respecto a los adultos mayores, que sí pueden verse afectados físicamente por la pandemia.

\section{Metodología}

Para llevar adelante esta investigación, se desarrolló un cuestionario único, para recolectar datos empíricos, que se envió aleatoriamente a través de diversas plataformas virtuales a seis universidades de Nariño en Colombia. El procedimiento de muestreo fue no probabilístico de conveniencia y bola de nieve. El enlace de esta encuesta se difundió, en primera instancia, entre seis profesores y 12 representantes estudiantiles adscritos a las siguientes instituciones de educación superior: Universidad Mariana, Universidad de Nariño, Universidad Cesmag, Corporación Universitaria Minuto de Dios y Corporación Universitaria Autónoma de Nariño. Las personas seleccionadas enviaron, a su vez, el enlace a sus compañeros. La encuesta estuvo disponible durante cuatro semanas entre el 25 de mayo y el 25 de junio de 2020.

Antes de aplicar el cuestionario definitivo, se realizó una prueba piloto que confirmó que los ítems se redactaron de manera comprensible y sin términos vagos, ambiguos o que pudieran ser malinterpretados; con lo que se elimina el sesgo de aquiescencia (Winkler, Kanouse y Ware, 1982) Para esto, se tradujeron las preguntas del idioma origi- 
nal (inglés y francés) manteniendo el ítem simple, pero sin perder la esencia. Al inicio de la encuesta se informó a los estudiantes el propósito de la investigación y, para abordar las posibles preocupaciones del sesgo de método común, se recurrió a las correcciones propuestas por Podsakoff, Mackenzie, Lee y Podsakoff (2003), entre las que se tuvo en cuenta controlar el efecto de coherencia y deseabilidad social (Crowne y Marlowe, 1964), para lo cual una misma persona no podía responder el instrumento más de una vez. Además, se hizo necesario proteger la privacidad de los encuestados; se les aseguró que no había respuestas correctas o incorrectas y que debían responder de la manera más honesta posible, y se les recordó que los datos recabados se utilizarían solo con fines de investigación.

La encuesta se conformó por cuatro apartados. El encuestado podía abandonar el cuestionario en el momento que lo quisiera y si deseaba, podía retomarlo, lo que garantizó así el consentimiento, el interés y la participación libre y voluntaria de los encuestados. El primer apartado contenía las preguntas sobre características sociales, demográficas y económicas; el segundo, sobre las motivaciones para estudiar; el tercero, acerca de los estados de ánimo; y el último, las preguntas relacionadas con situaciones vividas en la institución por consecuencia de la pandemia.

\subsection{Escalas empleadas}

Para medir las motivaciones, se tradujeron 20 ítems de la Échelle de Motivation en Éducation (EME) (Vallerand, Blais, Brière y Pelletier, 1989). Luego, cada ítem se puso a consideración de los expertos en las áreas de psicología y marketing, quienes hicieron observaciones a la correspondencia de los ítems con lo que se pretendía medir. Los expertos avalaron los 20 ítems que se tomaron de este cuestionario. Para medir estas motivaciones, se recurrió a la pregunta “Cuál es la importancia para ti de la siguiente afirmación en relación con tus estudios, en este momento?", a la cual le seguía la batería de motivaciones según la subdimensión evaluada (tabla 1). Estas razones fueron puntuadas de acuerdo con una escala tipo Likert de cinco puntos, desde 1: mucho menos importante, hasta 5: mucho más importante, con una puntuación intermedia 3 : medianamente importante.

Para medir los estados de ánimo se consideraron dos factores de la versión corta del perfil de estados de ánimo (POMS-SF, por sus siglas en inglés) propuesto por Curran, Andrykowski y Studts (1995), en atención al criterio de la Organización Mundial de la Salud (Papandrea, 2020) sobre los estados de ánimo más recurrentes en esta pandemia. La escala estaba compuesta por adjetivos que se configuraron para medir los estados de ánimo. Para ello, se recurrió a la pregunta “¿Hasta qué punto se te aplican las siguientes afirmaciones ahora mismo?", a la cual le seguía la batería de estados anímicos (tabla 1). Estos ítems se evaluaron con una escala tipo Likert de 5 puntos así: no se aplica en lo absoluto (1), no se aplica casi nada (2), medianamente aplica (3), algo se aplica (4) y se aplica mucho (5).
En el último apartado, para conocer la opinión de los estudiantes sobre las consecuencias de la pandemia y la posible relación con el marketing educativo, se formuló una serie de preguntas que luego de la revisión de literatura se incluyeron al definir la ausencia de instrumentos ajustados a esta realidad (tabla 3).

\subsection{Proceso de análisis}

Se procedió a una primera prueba piloto con dos grupos de estudiantes (80 en total) de las universidades analizadas para terminar con la validación estadística correspondiente, a partir de la cual se pudo comprobar la fiabilidad y la validez del cuestionario. Posterior a la aplicación, se procedió a medir el alpha de Cronbach global $(0,746)$, así como el mismo estadístico para cada uno de los factores en análisis: motivaciones (ítems del 13 al 32), cuyo resultado fue de 0,774; y estados de ánimo lítems del 33 al 39) con alpha de Cronbach de 0,805.

De los cuestionarios distribuidos, 685 fueron completados y devueltos. De este tamaño muestral, el 56,6\% pertenecía a la Universidad de Nariño; el 27,8\%, a la Universidad Mariana; el 8,4\%, a la Universidad Cesmag; el 3,7 y el 3,6\%, a las corporaciones Autónoma de Nariño y Minuto de Dios, respectivamente. El $62 \%$ de la población tenía entre 20 y 24 años, 60,7\% mujeres y 39,3\% hombres. En relación con el nivel académico, el $77,9 \%$ estudiaba pregrado y el restante, es decir, 22,1\%, posgrado. La mayor cantidad de participantes fue del área de ciencias sociales con un $40 \%$, seguida de ciencias humanas con un $30 \%$, ciencias agrícolas, ingeniería y ciencias de la salud cada una con un 15,10 y $5 \%$, respectivamente.

En esta investigación, se utilizaron dos técnicas estadísticas multivariantes. En la primera, se realizó un análisis factorial exploratorio tipo $\mathrm{R}$, con el uso del programa Statistical Package for the Social Sciences (SPSS) versión 22, debido a su practicidad para realizar este tipo de análisis, cuyo objetivo es "encontrar un modo de condensar la información contenida en un número de variables originales en un conjunto más pequeño de variables (factores) con una pérdida mínima de información" (Hair et al., 1999. p.11). Basados en dichos resultados, se aplicó un análisis de clústeres jerárquico, para encontrar una estructura de grupos homogénea. Para la selección de variables, se tomó el método deductivo, debido a que se tuvo en cuenta la literatura existente para tal fin, en este método se caracterizan los objetos que se están agrupando. Sobre el método de agrupación, se eligió el método jerárquico de Ward, en el que la distancia entre dos conglomerados es la suma de los cuadrados entre dos conglomerados sumados para todas las variables. Una vez definida la mejor solución en cuanto a número de grupos, se utilizó el método no jerárquico (Hair et al., 1999).

Después de realizar la división en clústeres, se describe cada uno bajo un cruce de los casos. Es importante informar que los análisis interdependientes buscan maximizar la explicación de la serie de variables entera, 
y no predecir dependencia de variables (Hair et al., 1999). En relación con el tamaño muestral para el uso de estos análisis, se cuenta con el número de observacio- nes a variables 18 a 1,dentro de los límites aceptables. Además, el tamaño muestral de 695 supone una base robusta para su cálculo.

Tabla 1. Resultados para la extracción de factores

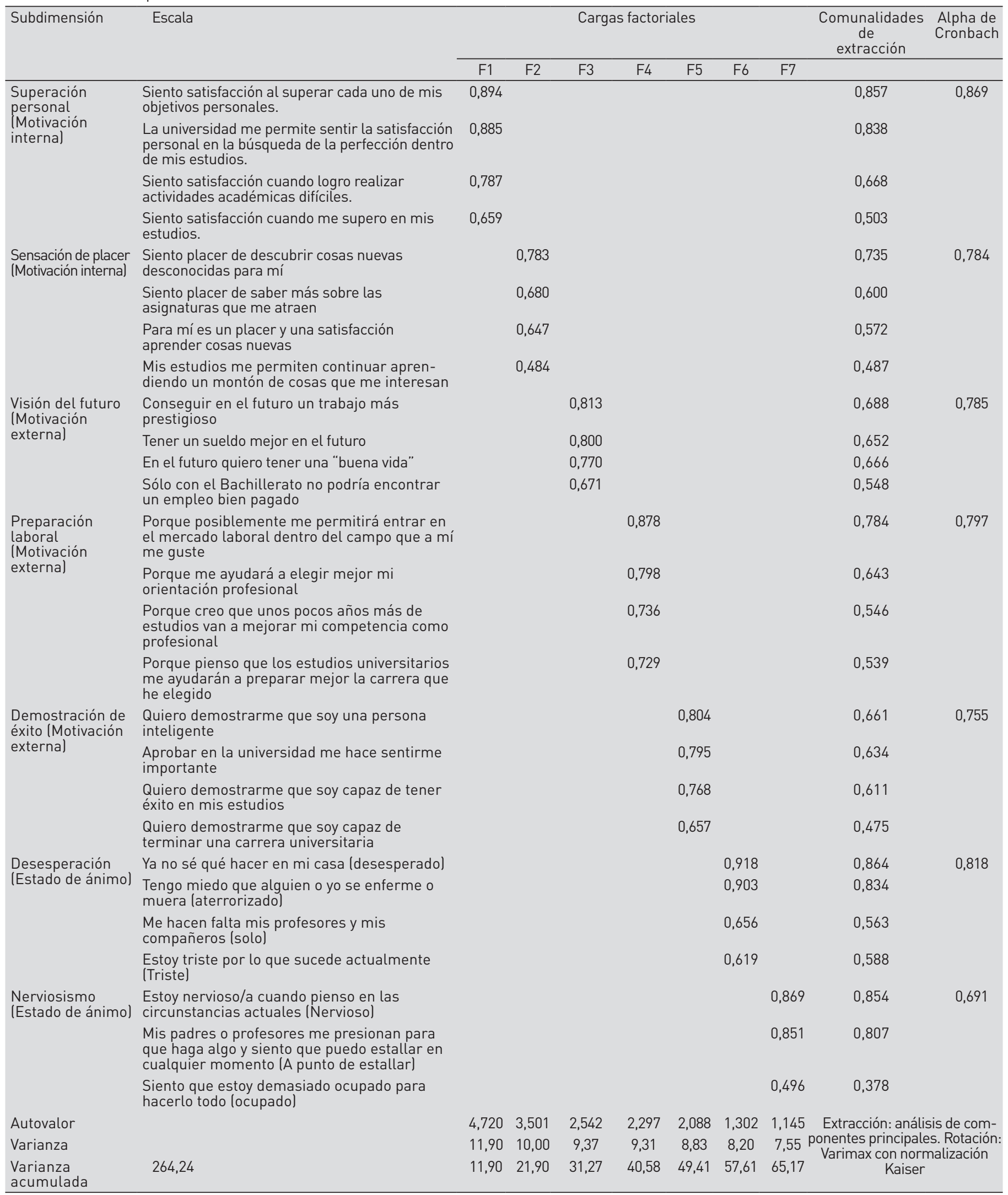

Fuente: elaboración propia. 


\section{Resultados}

A continuación, se presentan los resultados del análisis factorial propuesto. Luego de revisar el contraste de Bartlett, se observa en la muestra que las correlaciones no nulas existen $(\mathrm{p}<0,000$; chi-cuadrado $=10.263,26$; $\mathrm{gl}=351$ ) y el índice Kaiser Meyer y Olkin (KMO) (Kaiser, 1960), para comprobar que exista correlación adecuada entre las variables analizadas, fue de 0,712 , cuyo valor límite aceptable es de 0,70 (Hair et al., 1999). Cada variable excede el valor del umbral, lo que permitió el desarrollo de esta técnica. Por su parte, el número de factores definidos debe explicar al menos el $60 \%$ de la varianza para considerar un modelo adecuado (Malhotra y Dash, 2016) (tabla 1). Se utilizó un método de extracción de componentes principales, en el que se incluyeron solo aquellos factores cuyos coeficientes de autovalor son mayores a 1,0 (Hair et al., 1999).

Tanto para el análisis de las motivaciones como de los estados de ánimo, se tomó la decisión de conservar todos los ítems al corroborar que cumplían con las cargas necesarias para mantenerse dentro de la escala, pues tienen un valor mayor a 0,4 (Hair et al., 1999). Una vez realizadas las valoraciones de las escalas y obtener 7 factores para las dimensiones propuestas, se procedió a renombrar cada uno, al tomar como referencia las variables con mayores cargas para representar el factor. En el caso de las motivaciones, se mantienen las 5 subdimensiones propuestas por Ryan y Deci (2000) y se las renombra como 1) superación personal, en la que aparecen ítems relacionados con la satisfacción por el logro de objetivos; 2) sensación de placer, que cuenta con variables que tienen que ver con el gusto por aprender; 3) visión del futuro, acerca de que estudiar produce beneficios en el largo plazo; 4l preparación laboral, que hace referencia al estudio como un medio para lograr una mejor posición laboral; y 5) demostración de éxito, como una forma de dar a entender que es posible tener éxito en los estudios. Los resultados demuestran que, a pesar de ser en esencia diferentes, las motivaciones internas y externas no son antagónicas, sino que más bien coexisten con variaciones en cuanto a su intensidad y frecuencia (Avramova, Yaneva, y Bonev, 2014).

Por su parte, en relación con los estados de ánimo presentes en la pandemia, también fue posible conservar todos los ítems de la escala propuesta por Curran et al. (1995) y se optó por nombrar los dos factores obtenidos de la siguiente manera: 6) desesperación, que cuenta entre sus afirmaciones aquellas que valoran estados de ánimo negativos como el miedo, la soledad, la tristeza y la depresión; y 7) nerviosismo, como un estado de ánimo negativo relacionado con lo que sucede actualmente y la ansiedad por las preocupaciones propias de la incertidumbre que esto ha ocasionado. Estos resultados, como lo confirmó un estudio sobre estados de ánimo en Australia IVan Rheenen et al., 2020), revelan que la depresión, desesperación, miedo, soledad, tristeza, nervios, ansiedad, estrés y angustia en general se han elevado en situación de pandemia. También, una encuesta que se realizó en Estados
Unidos a finales de marzo de 2020 reveló que el 75\% de los estudiantes de educación superior respondieron que tienen niveles más altos de ansiedad, depresión y estrés (Goldrick-Rab et al., 2020).

Al final, se puede constatar que la escala explica el $65,17 \%$ de la varianza total acumulada, con una consistencia demostrada por el coeficiente alpha de Cronbach para cada factor igual a superación personal $=0,869$; sensación de placer $=0,784$; visión del futuro $=0,785$; preparación laboral $=0,797$; demostración del éxito $=0,755$; desesperación $=0,818$; y nerviosismo $=0,691$ (tabla 1).

Los factores obtenidos sobre motivaciones y estados de ánimo tienen relación con la intención por parte de los estudiantes de continuar con sus estudios (Covington y Omelich, 1984). No obstante, la comprobación de esta idea no es el objetivo de esta investigación. Sin embargo, se realizó un esfuerzo por indagar en las necesidades y los aspectos relacionados con el servicio que reciben los estudiantes para conectar estos estados psicológicos con el tema de marketing educativo y las implicaciones que ha tenido por causa de la pandemia por COVID-19. Para ello, con los resultados del análisis factorial, se realizó un análisis jerárquico de clústeres con los 7 factores obtenidos anteriormente. De acuerdo con la metodología propuesta, se pudo determinar que el número óptimo de clústeres es tres: el primero abarca los factores de superación personal y sensación de placer; el segundo, la desesperación, nerviosismo, preparación laboral y demostración de éxito; y el tercero, la visión de futuro (tabla 2 y figura 1).

Tabla 2. Tamaño de los clústeres

\begin{tabular}{lcc}
\hline Clúster & Casos & Porcentaje \\
\hline 1 & 263 & $37,8 \%$ \\
2 & 135 & $19,4 \%$ \\
3 & 297 & $42,7 \%$ \\
Total & 695 & $100 \%$ \\
\hline
\end{tabular}

Fuente: elaboración propia.

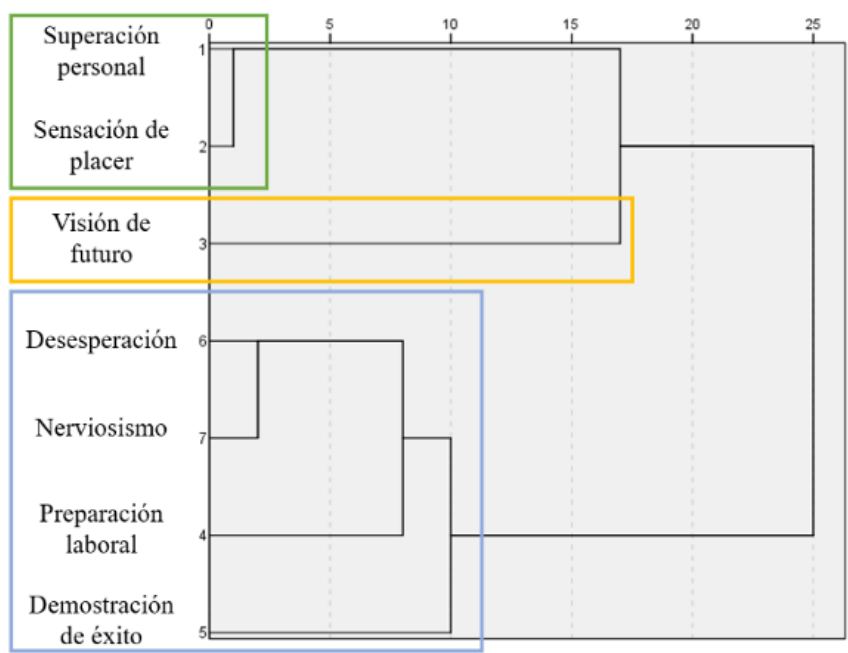

Figura 1. Dendograma de factores analizados Fuente: elaboración propia. 
Con esta información y las preguntas que relacionaban las variables de marketing educativo se agruparon los diferentes casos y se describieron los grupos tomando los valores más altos que resultaron para cada variable (tabla 3).

Clúster 1) los motivados: este grupo está formado por 263 individuos (37,8\%), en su mayoría mujeres de primeros semestres y de universidad privada, quienes están preocupadas por falta de espacios, problemas en los procesos de aprendizaje debido a la falta de contacto con los profesores, la posibilidad de disminuir precios en matrículas $y$ el aumento en sus responsabilidades familiares. Este grupo se identifica por estar motivado para continuar sus estudios, como parte importante de los objetivos personales, perfeccionamiento, superación, placer de aprender y conocer nuevas cosas que pueden llegar a interesarles.

Clúster 2) los trabajadores: representado por 135 individuos $(19,4 \%)$, en su mayoría estudiantes mujeres de primeros semestres, de universidad privada, preocupadas por la dificultad en las comunicaciones con la universidad y la falta de contacto con sus compañeros. Este grupo tiene interés en aprovechar sus estudios para ingresar en el mercado laboral y ser más competitivos, demostrar su inteligencia y sentirse importante por sus logros; sin embargo, los efectos de la pandemia los pone nerviosos y ansiosos a la vez.

Clúster 3) los futuristas: son el grupo más numeroso con 297 casos que representan el $42,7 \%$, en su mayoría hombres, de últimos semestres de universidad pública, quienes han señalado dificultades relacionadas con recursos informáticos e internet insuficientes y financiación para sostenimiento. Este clúster está determinado a estudiar para conseguir un futuro más prestigioso, representado por un buen ingreso que les permita darse una buena vida, ya que entienden que un título universitario es importante para lograrlo.

Sobre los descriptores, es importante asentar que se intentó relacionar cada variable del marketing mix de servicios con una pregunta para determinar la opinión de los estudiantes sobre esos aspectos. Sin embargo, para la variable producto, se realizó un ítem relacionado con la calificación que dieron los estudiantes sobre el manejo que las instituciones dieron a la pandemia, que puede asociarse con esta variable bajo la comprensión del producto como el servicio global. Los estudiantes respondieron con un $42 \%$ aceptable, seguido de buena con un 21\% (figura 2). Este ítem confirma lo revelado por Crawford et al. (2020), quienes afirman que los tipos de respuesta que se han llevado a cabo y la agilidad en su implementación no han sido los mejores; sin embargo, destacan que existen oportunidades importantes para aprender de los desarrollos de las experiencias significativas de otras universidades y fortalecer así la respuesta ante la COVID-19.

Si bien los estudiantes pueden reconocer las dificultades por las que atraviesan las universidades en este momento, existe una diversidad de estrategias digitales que se han venido implementando en lo que ha transcurrido del año por parte de las instituciones, las cuales han sido apoyadas por el Gobierno nacional (Crawford et al., 2020).

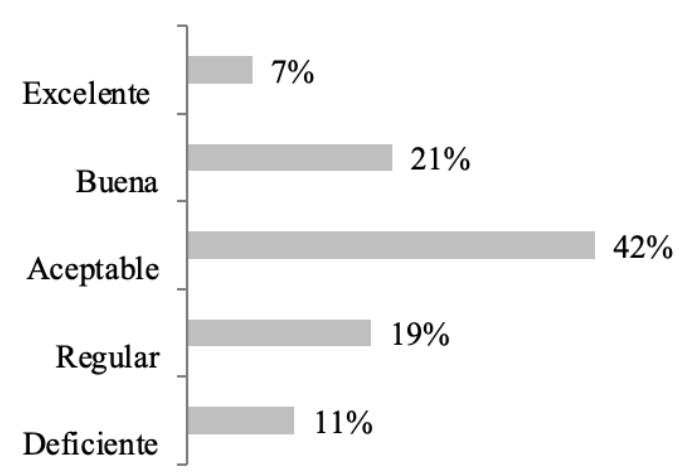

Figura 2. Calificación del manejo de la institución a la pandemia Fuente: elaboración propia.

\section{Conclusiones}

La pandemia por COVID-19 obligó a las personas a permanecer en sus hogares, debido a normas gubernamentales, y también exigió el cierre de las instituciones educativas en todos los niveles. Esto motivó a transitar de una modalidad presencial a una que estuviera mediada por las tecnologías, como estrategia para terminar el calendario académico propuesto a principio de año (García-Rey, 2020).

Aunque muchas organizaciones de educación superior se han centrado inicialmente en la transición al entorno virtual, ahora la atención se centra en la pedagogía en línea. Es posible comprender que probablemente no hubo un momento para una respuesta global coordinada, colaborativa y colectiva a los principios de las mejores prácticas para la instrucción en línea; sin embargo, quedan cuestionamientos y reflexiones muy importantes, ya que todo lo sucedido tiene el potencial de ser un facilitador de métodos digitales de educación más flexibles e innovadores, pero también podría conducir a menos actividades de aseguramiento de la calidad mientras el foco esté en la mitigación de ingresos y no en consolidar una propuesta pedagógica al respecto (Crawford et al., 2020).

Los resultados son acordes con investigaciones actuales, según las cuales la implementación tecnológica requiere considerar algunos elementos clave, a saber, es preciso que toda la comunidad educativa se encuentre preparada y motivada para el manejo de las tecnologías educativas, el diseño de entornos adecuados, la planificación y acción acorde al contexto virtual y las plataformas educativas con recursos y herramientas que permitan la entrega de un servicio de calidad (Videla, Vázquez-Cano, López-Meneses y Orrego, 2020). En este punto, resulta interesante retomar a Claxton (1990), quien proponía un modelo de "aprender a aprender", y que hoy en día resulta importante tener en cuenta, pues se busca una educación en la que toda la red educativa pueda realizar este proceso como parte de una planificación concertada y una red conformada por todos los actores involucrados, y en la que, por ende, ninguno de los actores se quede atrás, especialmente en casos de crisis, como los producidos por la pandemia. 
Un punto importante que deja esta investigación es que el paso a la educación mediada por tecnologías demanda una gran inversión en equipos, herramientas digitales y otros requerimientos propios para este formato educativo (Pedró, 2020), ya que los actores involucrados en el sistema educativo aún carecen de los estándares idóneos para la entrega de un buen servicio. En Colombia, se reportó que el $96 \%$ de los municipios tienen un déficit de recursos tecnológicos y 20 millones de habitantes carecen de banda ancha (Arias-Ortiz et al., 2020). Ante esto, el país ha realizado esfuerzos desde el Ministerio de Educación Nacional y de las Tecnologías de la Información y la Comunicación, con la donación de equipos y servicios de internet a lo largo del país en poblaciones con mayores dificultades.

También los gobiernos locales y las mismas universidades han tenido que hacer lo propio, entregando y apoyando a los estudiantes con préstamo de equipos y planes de datos para algunos estudiantes (MEN, 2020).

El precio tiene un impacto en dos sentidos; el primero al generar un "ahorro", por parte de las universidades, en algunos aspectos en la entrega del servicio, lo que permite una disminución en el costo de matrículas; el segundo, la complicada situación de los estudiantes para seguir pagando sus estudios. En este sentido y gracias a aportes del MEN, por medio del Fondo Solidario para la Educación, así como a los recursos dados por gobernaciones, alcaldías y las mismas universidades, se ha logrado la gratuidad para los estudiantes de 15 de las 32 universidades públicas en el país. Por su parte, las universidades privadas han diseñado sus propios programas de alivio para los estudiantes que más lo necesitan (Nieto, 2020).

Con respecto al nuevo espacio donde se presta el servicio, es decir, la casa del estudiante, es importante afirmar que el contacto familiar se amplió a raíz de la pandemia a las 24 horas del día. Las rutinas han sido un elemento clave para organizar el tiempo en este espacio, manteniendo horarios similares a los previos a la pandemia; sin embargo, las actividades en el hogar aumentaron para cada integrante, lo que dificulta claramente el desarrollo de algunas de ellas, incluido el estudio (Serrano-Cumplido et al., 2020).

Las personas en los servicios son probablemente una de las preocupaciones más importantes para el marketing educativo, por ello los procesos de capacitación periódica resultan relevantes para todos los grupos de interés. Además, las universidades están llamadas a implementar redes educativas en línea para todos los interesados; esto permite que poco a poco quienes estaban menos acostumbrados a este formato educativo opten por la solución que mejor se ajuste a sus necesidades (Orr et al., 2020). Sin embargo, el diálogo y la construcción del conocimiento cara a cara es irreemplazable.

Por su parte, las comunicaciones han sido consideradas como un servicio esencial en esta crisisyse puso en evidencia la falta de estructuras y uso correcto de ellas (Cerezo, 2020) en las instituciones de educación, que no logran aprovechar todos los medios que están a disposición para conectar con los estudiantes. Es importante que todas las instituciones se fortalezcan en esta variable a la hora de ofertar y enganchar con quienes fueron, son y serán sus futuros clientes. Por lo pronto, resulta determinante enfocarse en la generación de confianza y en el cuidado de la salud como temas prioritarios por atender.

Tabla 3. Descriptores de los grupos

\begin{tabular}{|c|c|c|c|c|}
\hline Variable sugerida & Interrogante & Clúster 1 & Clúster 2 & Clúster 3 \\
\hline \multirow[t]{2}{*}{ Precio } & ¿Esperarías que el precio de la matricula disminuya? & $37,8 \%$ & $19,42 \%$ & $42,7 \%$ \\
\hline & $\begin{array}{l}\text { ¿Has recibido algún tipo de financiación para tu } \\
\text { semestre? }\end{array}$ & $37,7 \%$ & $17,75 \%$ & $44,6 \%$ \\
\hline \multirow[t]{2}{*}{ Prueba física } & $\begin{array}{l}\text { La biblioteca cerrada o sus recursos, ¿te impiden } \\
\text { seguir tus estudios? }\end{array}$ & $37,4 \%$ & $19,57 \%$ & $43,0 \%$ \\
\hline & $\begin{array}{l}\text { Recursos informáticos o internet insuficiente, ¿te impi- } \\
\text { den seguir tus estudios? }\end{array}$ & $36,6 \%$ & $18,07 \%$ & $45,4 \%$ \\
\hline Personal & $\begin{array}{l}\text { La falta de contacto compañeros, ¿te impiden seguir tus } \\
\text { estudios? }\end{array}$ & $38,3 \%$ & $20,85 \%$ & $40,9 \%$ \\
\hline Personal & ¿Han aumentado tus responsabilidades familiares? & $40,4 \%$ & $19,26 \%$ & $40,4 \%$ \\
\hline Procesos & $\begin{array}{l}\text { La falta de contacto con el profesor, ¿dificulta tu apren- } \\
\text { dizaje? }\end{array}$ & $40,4 \%$ & $20,87 \%$ & $38,8 \%$ \\
\hline \multirow[t]{2}{*}{ Género } & Mujeres & $38,6 \%$ & $19,43 \%$ & $41,9 \%$ \\
\hline & Hombres & $36,6 \%$ & $19,41 \%$ & $44,0 \%$ \\
\hline \multirow[t]{2}{*}{ Tipo de universidad } & Universidad Privada & $40,1 \%$ & $20,5 \%$ & $39,4 \%$ \\
\hline & Universidad Pública & $36,1 \%$ & $18,6 \%$ & $45,3 \%$ \\
\hline
\end{tabular}

Fuente: elaboración propia. 
Esta investigación también deja en evidencia la importancia de conocer las motivaciones de los estudiantes para aprender. Sobre estas, las universidades deberían responder proactivamente con la creación de estímulos que los apoyen en su idea de continuar sus estudios (Papandrea, 2020). Además, el análisis de los estados de ánimo permite la inclusión en la promesa de valor de ayudas psicoemocionales a través de sesiones o programas en línea que permitan tratar estos temas, y que faciliten una respuesta oportuna y adecuada a los estudiantes que más lo necesitan (Skilbeck, 2002). Esto permite reiterar la importancia de ver el marketing no desde la visión comercial, sino como una ayuda para responder a estos sucesos de manera acorde y congruente con la realidad y el contexto (Vargo y Lusch, 2004).

Entre las limitaciones de este tipo de estudios y bajo el contexto de la pandemia, se puede mencionar la dificultad de contar con escalas apropiadas y contextualizadas. Además, la información sobre lo que está sucediendo trastoca mucho las posiciones de los estudiantes; por tanto, se sugiere que una vez terminados todos estos acontecimientos se realicen investigaciones que permitan evaluar las dimensiones tratadas en este artículo y otras con el fin de contrastarlas y ampliarlas.

\section{Agradecimientos}

A docentes, representantes estudiantiles y estudiantes que hicieron posible el desarrollo de esta investigación. ¡Gracias y a seguir adelante!

\section{Conflicto de intereses}

Los autores declaran no tener ningún conflicto de intereses.

\section{Bibliografía}

American Marketing Association (2017). Definition of marketing. Recuperado el 12 de diciembre de 2020, de: https://www.ama. org/the-definition-of-marketing-what-is-marketing/

Ames, R. y Ames, C. (1984). Research on motivation in education: Student motivation. Orlando: Academic Press.

Arias-Ortiz, E., Rieble-Aubourg, S., Álvarez-Marinelli, H., Rivera, M. C., Viteri, A., López, A., ... y Scannone, R. (2020). La educación en tiempos del coronavirus: los sistemas educativos de América Latina y el Caribe ante COVID-19. Banco Interamericano de Desarrollo. https://doi.org/10.18235/0002337

Asociación Colombiana de Universidades (2020). Análisis breve de cifras de educación superior en Colombia. Recuperado el 12 de diciembre de 2020, de: https://www.ascun.org.co/noticias/ detalle/analisis-breve-de-cifras-de-educacion-superior-encolombia

Avramova, N., Yaneva, K. y Bonev, B. (2014). First-year dental students' motivation and attitudes for choosing the dental profession. Acta Medica Académica, 43(2), 113-121.

https://doi.org/10.5644/ama2006-124.110

Bagozzi, R. P., Gopinath, M. y Nyer, P. U. (1999). The role of emotions in marketing. Journal of the academy of marketing science, 27(2), 184-206. https://doi.org/10.1177/0092070399272005

Booms, B. H. y Bitner, M. (1981). Marketing strategies and organization structure for service firms. En J.H. Donelly y W. R. George (Eds.),
Marketing of Services (pp. 47-51). Chicago: American Marketing Association.

Brophy, J. (1983). Conceptualizing student motivation. Educational psychologist, 18(3), 200-215. https://doi.org/10.1080/00461528309529274

Cabrera, J. (2020). Efectos del coronavirus en el sistema de enseñanza: aumenta la desigualdad de oportunidades educativas en España. Revista de Sociología de la Educación, 13(2), 114-139. https://doi.org/10.7203/RASE.13.2.17125

Cerezo, P. (2020). El impacto de la pandemia en la prensa. Recuperado el 12 de diciembre de 2020, de: http://evocaimagen.com/dosieres/ dosier-evoca-09-medios-y-coronavirus.pdf

Charles, N. (2020). Increased mood disorder symptoms, perceived stress, and alcohol use among college students during the COVID-19 pandemic. https://doi.org/10.31234/osf.io/rge9k

Claxton, G. (1990). Teaching to learn: A direction for education. London: Cassell.

Covington, M. V. y Omelich, C. L. (1984). Task-oriented versus competitive learning structures: Motivational and performance consequences. Journal of Educational psychology, 76(6), 1038-1050. https://doi.org/10.1037/0022-0663.76.6.1038

Crawford, J., Butler-Henderson, K., Rudolph, J., Malkawi, B., Glowatz, M., Burton, R., ... y Lam, S. (2020). COVID-19: 20 countries' higher education intra-period digital pedagogy responses. Journal of Applied Learning \& Teaching, 3(1), 1-20. https://doi.org/10.37074/jalt.2020.3.1.7

Crowne, D. P. y Marlowe, D. (1964). The approval motive: Studies in evaluative dependence. New York: Wiley.

Curran, S. L., Andrykowski, M. A. y Studts, J. L. (1995). Short form of the profile of mood states (POMS-SF): Psychometric information. Psychological Assessment, 7(1), 80-83. https://doi.org/10.1037/1040-3590.7.1.80

Deci, E. L. y Ryan, R. M. (1985). Self-determination in human behavior. New York: Plenum Press.

Del Rio, C. y Malani, P. N. (2020). 2019 novel coronavirus-important information for clinicians. JAMA, 323(11), 1039-1040. https://doi.org/10.1001/jama.2020.1490

Dorn, E., Hancock, B., Sarakatsannis, J. y Viruleg, E. (2020). COVID-19 and student learning in the United States: The hurt could last a lifetime. McKinsey \& Company. Recuperado el 12 de diciembre de 2020, de: https://n9.cl/etail

Forgas, J. P. y Bower, G. H. (1987). Mood effects on person-perception judgments. Journal of Personality and Social Psychology, 53(1), 53-60. https://doi.org/10.1037/0022-3514.53.1.53

Frijda, N. H. (1986). The emotions. Cambridge, UK: Cambridge University Press.

García-Rey, T. (2020). Impacto del COVID-19 en la educación. Acta de Otorrinolaringología \& Cirugía de Cabeza y Cuello, 48(2), 131-132.

Goldrick-Rab, S., Coca, V., Kienzl, G., Welton, C., Dahl, S. y Magnelia, S. (2020). New evidence on basic needs insecurity and student wellbeing. Hope Center. Recuperado el 12 de diciembre de 2020, de: https://n9.cl/bq95

Goodell, J. W. (2020). COVID-19 and finance: Agendas for future research. Finance Research Letters, 35, 101512. https://doi.org/10.1016/j.frl.2020.101512

Grönroos, C. (1982). An applied service marketing theory. European Journal of Marketing, 16(7), 30-41. https://doi.org/10.1108/EUM0000000004859

Hair, J. F., Anderson, R. E., Tatham, R. L. y Black, W. C. (1999). Análisis multivariante. Madrid: Prentice Hall.

Harvey, J. A. (1996). Marketing schools and consumer choice. International Journal of Educational Management, 10(4), 26-32. https://doi.org/10.1108/09513549610122165

Hernández, M. O. (2020). A causa de la COVID-19... ¿perdimos el curso escolar. Mendive. Revista de Educación, 18(3), 452-456.

Hylén, J. (2006). Open educational resources: Opportunities and challenges. Proceedings of open education. Recuperado el 12 de diciembre de 2020, de: http://www.oecd.org/education/ceri/37351085.pdf

Idrovo, A. J. (2020). Primeras lecciones de la pandemia de COVID-19 a la educación médica colombiana. Revista Salud UIS, 52(2), 87-88. https://doi.org/10.18273/revsal.v52n2-2020002 
Kaiser, H. F. (1960). The application of electronic computers to factor analysis. Educational and Psychological Measurement, 20, 141- 151.

Malhotra, N. K. y Dash, S. (2016). Marketing research: An applied orientation. Londres: Pearson.

Maslow, A. (1954). Motivación y personalidad: la jerarquía de las necesidades básicas. Madrid España: Ediciones Díaz de Santos S. A

McCarthy, E. J. (1960). Basic marketing: A managerial approach. Homewood: R. D. Irwin.

Mead, G. H. (1934). Mind, self and society. Chicago: University of Chicago Press.

Miller, N.E. y Dollard, J.C. (1941). Social Learning and Imitation. New Haven: Yale University Press.

Morgan, C. T. (1943). Physiological Psychology. New York: McGraw-Hill.

Nieto, J. (23 de agosto de 2020). Matrícula cero: estos son los descuentos en las universidades públicas. El Tiempo. Recuperado el 12 de diciembre de 2020, de: https://n9.cl/ipmwo

Organización de las Naciones Unidas para la Educación, la Ciencia y la Cultura- Unesco. (2020a). Consecuencias negativas del cierre de las escuelas. Recuperado el 12 de diciembre de 2020, de: https://es.unesco.org/news/unesco-agrupa-organizacionesinternacionales-sociedad-civil-y-sector-privado-ampliacoalicion

Organización de las Naciones Unidas para la Educación, la Ciencia y la Cultura- Unesco. (2020b). Surgen alarmantes brechas digitales en el aprendizaje a distancia. Recuperado el 12 de diciembre de 2020 , de: https://es.unesco.org/news/surgen-alarmantes-brechasdigitales-aprendizaje-distancia

Orr, D., Luebcke, M., Schmidt, J. P., Ebner, M., Wannemacher, K., Ebner, M. y Dohmen, D. (2020). From lines of development to scenarios. En O. Dominic, M. Luebcke, J. P. Schmidt, M. Ebner, K. Wannemacher, M. Ebner y D. Dohmen (Eds.), Higher Education Landscape 2030 (pp. 5-24). Cham: Springer https://doi.org/10.1007/978-3-030-44897-4_2

Papandrea, D. (2020). In the face of a pandemic: Ensuring safety and health at work. Geneva: ILO.

Pedró, F. (2020). COVID-19 y educación superior en América Latina y el Caribe: efectos, impactos y recomendaciones políticas. Análisis Carolina, (36), 12-15.

Podsakoff, P. M., MacKenzie, S. B., Lee, J.-Y. y Podsakoff, N. P. (2003). Common method biases in behavioral research: A critical review of the literature and recommended remedies. Journal of Applied Psychology, 88(5), 879-903. https://doi.org/10.1037/0021-9010.88.5.879

República de Colombia, Ministerio de Educación Nacional (14 de marzo de 2020). Circular 19. Orientaciones con ocasión a la declaración de emergencia sanitaria provocada por el coronavirus COVID-19. Bogotá, Colombia.

República de Colombia, Ministerio de Salud y Protección Social (12 de marzo de 2020). Por la cual se declara la emergencia sanitaria por causa del coronavirus COVID-19 y se adoptan medidas para hacer frente al virus. [Resolución 385 de 2020]. DO 51.284. Bogotá.

Rushton, A. M. y Carson, D. J. (1985). The marketing of services: Managing the intangibles. European Journal of Marketing, 19(3), 19-40. https://doi.org/10.1108/EUM0000000000582

Ryan, R. M. y Deci, E. L. (2000). La teoría de la autodeterminación y la facilitación de la motivación intrínseca, el desarrollo social, y el bienestar. American Psychologist, 55(1), 68-78. https://doi.org/10.1037110003-066X.55.1.68

Serrano-Cumplido, A., Ortega, P. A. E., García, A. R., Quintana, V. O., Fragoso, A. S., Garcia, A. B. y Bayón, Á. M. (2020). COVID-19. La historia se repite y seguimos tropezando con la misma piedra. Medicina de Familia. SEMERGEN.

Shostack, G. L. (1982). How to design a service. European Journal of Marketing, 16(1), 49-63. https://doi.org/10.1108/EUM0000000004799

Skilbeck, M. (2002). The university challenged: A review of international trends and issues with particular relevance to Ireland. Dublin: Higher Education.

Sohrabi, C., Alsafi, Z., O'Neill, N., Khan, M., Kerwan, A., Al-Jabir, A., ... y Agha, R. (2020). World Health Organization declares global emergency: A review of the 2019 novel coronavirus (COVID-19). International Journal of Surgery, 76, 71-76. https://doi.org/10.1016/j.ijsu.2020.02.034

Speth, M. M., Singer-Cornelius, T., Oberle, M., Gengler, I., Brockmeier, S. J. y Sedaghat, A. R. (2020). Mood, anxiety and olfactory dysfunction in COVID-19: Evidence of central nervous system involvement? The Laryngoscope, 130(11), 2520-2525. https://doi.org/10.1002/lary.28964

Taylor, C.R (2020). Advertising and COVID-19. International Journal of Advertising, 39(5), 587-589. https://doi.org/10.1080/02650487.2020.1774131

Thyer, B. A. (1987). Treating anxiety disorders: A guide for human service professionals. London: Sage Publications.

Ting, D. S. W., Carin, L., Dzau, V. y Wong, T. Y. (2020). Digital technology and COVID-19. Nature medicine, 26(4), 459-461. https://doi.org/10.1038/s41591-020-0824-5

Toledo, A. S. y Armas, N. (2020). TIPS de marketing en épocas de COVID y Post COVID. CienciAmérica, 9(2), 99-108. http://dx.doi.org/10.33210/ca.v9i2.300

Vallerand, R. J., Blais, M. R., Brière, N. M. y Pelletier, L. G. (1989). Construction et validation de l'échelle de motivation en éducation (EME). Canadian Journal of Behavioural Science/Revue canadienne des Sciences du Comportement, 21(3), 323-349. https://doi.org/10.1037/h0079855

Van Rheenen, T. E., Meyer, D., Neill, E., Phillipou, A., Tan, E. J., Toh, W. L. y Rossell, S. L. (2020). Mental health status of individuals with a mood-disorder during the COVID-19 Pandemic in Australia: Initial results from the COLLATE Project: COVID-19 and mood disorders. Journal of Affective Disorders, 275, 69-77. https://doi.org/10.1016/j.jad.2020.06.037

Vargo, S. L. y Lusch, R. F. (2004). Evolving to a new dominant logic for marketing. Journal of Marketing, 68(1), 1-17. https://doi.org/10.1509/jmkg.68.1.1.24036

Videla, C. B., Vázquez-Cano, E., López-Meneses, E. y Orrego, R. A. (2020). Proyecto Difpronet: análisis de las dificultades, propuestas y retos educativos ante el COVID-19. IJERI: International Journal of Educational Research and Innovation, (15), 17-34. https://doi.org/10.46661/ijeri.5145

Wang, C., Cheng, Z., Yue, X. G. y McAleer, M. (2020). Risk management of COVID-19 by universities in China. Journal of Risk and Financial Management, 13(2), 36. https://doi.org/10.3390/jrfm13020036

Winkler, J. D., Kanouse, D. E. y Ware, J. E. (1982). Controlling for acquiescence response set in scale development. Journal of Applied Psychology, 67(5), 555-561. https://doi.org/10.1037/0021-9010.67.5.555

Zhang, J., Shuai, L., Yu, H., Wang, Z., Qiu, M., Lu, L., ... y Chen, R. (2020). Acute stress, behavioural symptoms and mood states among school-age children with attention-deficit/hyperactive disorder during the COVID-19 outbreak. Asian Journal of Psychiatry, 51, 102077. https://doi.org/10.1016/j.ajp.2020.102077 